\title{
A Cutting Process for Random Mappings
}

\author{
Jennie C. Hansen and Jerzy Jaworski
}

\begin{abstract}
In this paper we consider a cutting process for random mappings. Specifically, for $0<m<n$, we consider the initial (uniform) random mapping digraph $G_{n}$ on $n$ labelled vertices, and we delete (if possible), uniformly and at random, $m$ non-cyclic directed edges from $G_{n}$. The maximal random digraph consisting of the uni-cyclic components obtained after cutting the $m$ edges is called the trimmed random mapping and is denoted by $G_{n}^{m}$. If the number of non-cyclic directed edges is less than $m$, then $G_{n}^{m}$ consists of the cycles, including loops, of the initial mapping $G_{n}$. We consider the component structure of the trimmed mapping $G_{n}^{m}$. In particular, using the exact distribution we determine the asymptotic distribution of the size of a typical random connected component of $G_{n}^{m}$ as $n, m \rightarrow \infty$. This asymptotic distribution depends on the relationship between $n$ and $m$ and we show that there are three distinct cases: (i) $m=o(\sqrt{n})$, (ii) $m=\beta \sqrt{n}$, where $\beta>0$ is a fixed parameter, and (iii) $\sqrt{n}=o(m)$. This allows us to study the joint distribution of the order statistics of the normalized component sizes of $G_{n}^{m}$. When $m=o(\sqrt{n})$, we obtain the Poisson$\operatorname{Dirichlet}(1 / 2)$ distribution in the limit, whereas when $\sqrt{n}=o(m)$ the limiting distribution is Poisson-Dirichlet(1). Convergence to the Poisson-Dirichlet $(\theta)$ distribution breaks down when $m=O(\sqrt{n})$, and in particular, there is no smooth transition from the $\mathcal{P} \mathcal{D}(1 / 2)$ distribution to the $\mathcal{P} \mathcal{D}(1)$ via the Poisson-Dirichlet distribution as the number of edges cut increases relative to $n$, the number of vertices in $G_{n}$.
\end{abstract}

\section{Introduction}

In this paper we consider the component structure of a trimmed random mapping. Informally, we start with a uniform random mapping from the 
vertices $V_{n}=\{1,2, \ldots, n\}$ into $V_{n}$. Any such mapping can be represented as a directed graph on $n$ labelled vertices which has components consisting of directed cycles with directed trees attached. We 'trim' the trees in the random mapping graph by selecting and deleting a number of tree edges at random. This cutting procedure gives rise to a directed graph consisting of uni-cyclic components (these correspond to the original components of the random mapping) and tree components which result from the cutting procedure. We discard the tree components and call the remaining graph the trimmed random mapping. In this paper we consider the distribution of the component sizes in the trimmed random mapping as a function of the number of edges cut. Before discussing the motivation for this investigation, we introduce some notation and review well-known asymptotic results for the component structure of the uniform random mapping.

For $n \geq 1$, let $\mathcal{M}_{n}$ denote the set of mappings $f: V_{n} \rightarrow V_{n}$, and let $\mathcal{T}_{n}$ denote the uniform random mapping of $V_{n}$ into $V_{n}$ with distribution given by

$$
\operatorname{Pr}\left\{\mathcal{T}_{n}=f\right\}=\frac{1}{n^{n}}
$$

for each $f \in \mathcal{M}_{n}$. The random mapping $\mathcal{T}_{n}$ can be represented by a directed random graph $G_{n}$ on vertices labelled $1,2, \ldots, n$, such that a directed edge from vertex $i$ to vertex $j$ exists in $G_{n}$ if and only if $\mathcal{T}_{n}(i)=j$. Since each vertex in $G_{n}$ has out-degree 1 , the components of $G_{n}$ consist of directed cycles with directed trees attached.

Much is known (see for example the monograph by Kolchin [33]) about the component structure of the random digraph $G_{n}$ which represents $\mathcal{T}_{n}$. Aldous [2] has shown that the joint distribution of the normalized order statistics for the component sizes in $G_{n}$ converges, as $n \rightarrow \infty$, to the Poisson-Dirichlet $(\theta)$ distribution with parameter $\theta=1 / 2$, which we denote by $\mathcal{P} \mathcal{D}(1 / 2)$, on the simplex

$$
\nabla=\left\{\left\{x_{i}\right\}: \sum x_{i} \leq 1, x_{i} \geq x_{i+1} \geq 0 \text { for every } i \geq 1\right\}
$$

as $n \rightarrow \infty$. Also, if $N_{k}$ denotes the number of components of size $k$ in $G_{n}$ then the joint distribution of $\left(N_{1}, N_{2}, \ldots, N_{b}\right)$ is close, in the sense of total variation, to the joint distribution of a sequence of independent Poisson random variables when $b=o(n / \log n)$ (see Arratia et.al. [6], [7]) and from this result one obtains a functional central limit theorem for the component sizes (see also [15]). The asymptotic distributions of variables such as the 
number of predecessors and the number of successors of a vertex in $G_{n}$ are also known (see [10, 40, 29, 30]). It is also known that embedded in every uniform random mapping there is a uniform random permutation. Specifically, for $n \geq 1$, let $L_{n}$ denote the number of cyclic vertices in $G_{n}$, where $i \in V_{n}$ is a cyclic vertex of $G_{n}$ if and only if there is some $k \geq 1$ such that $\mathcal{T}_{n}^{(k)}(i)=$ $i$. Then, given $L_{n}=l$, the random mapping $\mathcal{T}_{n}$ restricted to $\mathcal{L}_{n}$, the set of cyclic vertices of $G_{n}$, is a uniformly distributed random permutation on the $\ell$ vertices in $\mathcal{L}_{n}$. The cycle structure of uniform random permutations is well understood. In particular, the joint distribution of the normalized order statistics for the cycle lengths of a uniform random permutation also converges to the $\mathcal{P} \mathcal{D}(\theta)$ distribution on $\nabla([42])$, but in this case $\theta=1$.

Uniform random mappings and uniform random permutations are just two examples of random combinatorial structures where the $\mathcal{P} \mathcal{D}(\theta)$ distribution arises naturally as the limiting distribution for the order statistics of the normalized 'component' sizes of the structure. Other examples include, with $\theta=1$, prime factorisation of integers $([13])$, factorisation of polynomials over finite fields ([17]), and factorisation of matrices over finite fields ([16]), and, with $\theta=1 / 2$, uniform mapping patterns ([34]), bipartite random mappings ([19]), certain non-uniform random mappings ([3], [4]), and Poisson compound random mappings $([20])$. It is also possible to generate examples where the $\mathcal{P} \mathcal{D}(\theta)$ distribution with arbitrary parameter $\theta>0$ arises as a limiting distribution (see [8]), but these examples are somewhat artificial. For example, one can consider a random permutation $\sigma_{n}^{\theta}$ on $[n]=\{1,2, \ldots, n\}$ where the distribution of the cycle structure of $\sigma_{n}^{\theta}$ is given by the Ewens sampling formula with parameter $\theta>0$, and we note that when $\theta=1, \sigma_{n}^{\theta}$ is just the usual uniform random permutation on $[n]$. Then as $n \rightarrow \infty$, the joint distribution of the normalized order statistics of the cycle sizes of $\sigma_{n}^{\theta}$ converges to the $\mathcal{P} \mathcal{D}(\theta)$ distribution. This example is artificial in the sense that the limiting $\mathcal{P} \mathcal{D}(\theta)$ distribution is 'pre-determined' by correctly choosing the distribution for $\sigma_{n}^{\theta}$.

The trimmed random mapping model considered in this paper is a random structure which is (in some sense) sandwiched between a uniform random mapping and a uniform random permutation. Specifically, if no edges are cut, we have a uniform random mapping, whereas if all the trees are trimmed down to their roots, we have a random permutation on the root vertices. In light of this observation, one might suppose that if $m(n)$ edges are cut, where $m(n) \rightarrow \infty$ as $n \rightarrow \infty$, then the joint distribution of the normalized order statistics of the component sizes of the resulting trimmed random mapping 
converges to the $\mathcal{P} \mathcal{D}(\theta)$ distribution with parameter $1 / 2<\theta<1$ (where the value of $\theta$ may depend on how $m(n)$ goes to infinity). In this paper we show that in fact something quite different happens. More precisely, we show that if $m(n)=o(\sqrt{n})$ then we obtain a $\mathcal{P} \mathcal{D}(1 / 2)$ distribution in the limit, whereas for $\sqrt{n}=o(m(n))$ we obtain the $\mathcal{P} \mathcal{D}(1)$ distribution in the limit. There is a 'phase transition' when $m(n)=\beta \sqrt{n}$, where $\beta>0$ is a fixed parameter, and in this case we show that the limiting distribution cannot be $\mathcal{P} \mathcal{D}(\theta)$.

We note that our investigation of trimmed random mappings is close in spirit to the study of the evolution of the random mapping $\left(T_{n} ; q\right)$ and the corresponding random graph process (see [25], [26], [27], [28]). In fact, the "evolution" parameter $q$, which corresponds to the probability of a loop at a vertex, can be treated as the parameter which determines the number of edges (roughly $\lfloor n q\rfloor$ ) removed from the digraph representing a uniform random mapping (see [27]). In light of related results for $\left(T_{n} ; q\right)$, the phase transition which we have identified when $O(\sqrt{n})$ non-cyclic edges are cut in a random mapping is not very surprising. There has also been much work, initiated by Meir and Moon in 1970 [35], on 'cutting down' uniform random trees (forests) on $n$ vertices. Meir and Moon gave very precise asymptotic formulas for mean and variance of the number of edges that must be removed before isolating the roots, and again this number turns to be of order $\sqrt{n}$. For the most recent results in this direction see Janson [23].

In another direction, the structure of the random forest created by cutting edges in a uniform tree on $n$ vertices has been studied in detail (see especially [5], [11], [38]) and a 'phase transition' identified when $O(\sqrt{n})$ edges are cut, as well. In addition, in the case when $\beta \sqrt{n}$ edges are cut, the asymptotic joint distribution of the normalised sizes of the trees has been characterised in terms of the jumps of a stable $1 / 2$ subordinator $S_{t}$ on the interval $[0, \beta]$, conditioned on $S_{\beta}=1$. It would be interesting to investigate further connections between our results for trimmed random mappings and above results for trees and forests.

The paper is organized as follows. In Section 2 we carefully define the cutting process for uniform random mappings and establish some basic lemmas. In Section 3 we give a characterization of the $\mathcal{P} \mathcal{D}(\theta)$ distribution and describe a method for determining convergence to the $\mathcal{P} \mathcal{D}(\theta)$ distribution. In Section 4, using the exact distribution, we study the asymptotic distribution of the size of a typical component after cutting $m(n)$ edges, where $m(n) \rightarrow \infty$ as $n \rightarrow \infty$, as well as we considering the asymptotic joint distribution of the normalized order statistics of the sizes of the components of a 
trimmed random mapping.

Finally, throughout this paper we adopt the following abuse of notation: Suppose that $0<x<\infty$ is fixed and $n \in Z^{+}$, then by 'integer $m=x f(n)^{\text {', }}$ where $f$ is a function of $Z^{+}$, we mean $m=\lfloor x f(n)\rfloor$. Likewise, if $X$ is an integer-valued random variable, by ' $X=x f(n)$ ' we mean $X=\lfloor x f(n)\rfloor$.

\section{Trimmed Random Mappings}

In this section we define the trimmed random mapping $\mathcal{T}_{n}^{m}$ in terms of the random digraph $G_{n}^{m}$ which represents the action of $\mathcal{T}_{n}^{m}$ on a (random) set of vertices. To construct the random digraph $G_{n}^{m}$ for $1 \leq m \leq n$, we start with the random digraph $G_{n}$ which represents the uniform random mapping $\mathcal{T}_{n}$ on $V_{n}$ and we select (if it is possible) $m$ (directed) edges from all edges in $G_{n}$ which are not part of a cycle in $G_{n}$ such that any such subset of $m$ edges is equally likely to be selected. The $m$ selected edges are deleted from $G_{n}$ to create a random digraph $D_{n}^{m}$ on the vertices $V_{n}$ which consists of directed trees and uni-cyclic components. If the number of non-cyclic edges in $G_{n}$ is less than $m$, then we delete all non-cyclic edges to obtain $D_{n}^{m}$, which in this case consists of the cycles of the initial digraph $G_{n}$ and isolated vertices which correspond to the non-cyclic vertices of $G_{n}$. In all cases we let $G_{n}^{m}$ denote the maximal random directed subgraph of $D_{n}^{m}$ which consists of the uni-cyclic components of $D_{n}^{m}$. We also let $V_{n}^{m}$ denote the (random) vertex set of $G_{n}^{m}$ and let $\mathcal{T}_{n}^{m}$ denote the random mapping on the $V_{n}^{m}$ which corresponds to the random digraph $G_{n}^{m}$. Finally, we note that every component of $G_{n}^{m}$ is a 'remnant' of some component of $G_{n}$ which has been 'trimmed' and the cyclic vertices of $G_{n}^{m}$ are exactly the cyclic vertices of the original digraph $G_{n}$.

We define $\nu_{n}(m) \equiv\left|V_{n}^{m}\right|$ and $t_{n}(m)$ to be the size of the random rooted forest (with $m$ roots) which is created after cutting $m$ edges from the initial random mapping digraph $G_{n}$ (as noted above, this forest may consist of only $m$ roots). It is clear from the description of the cutting phase and the definition of $V_{n}^{m}$ that

$$
t_{n}(m)=n-\nu_{n}(m) .
$$

As a first step in our investigation of the component structure of the trimmed mapping digraph $G_{n}^{m}$, we determine the distribution of $t_{n}(m)$ (and hence, the distribution of $\left.\nu_{n}(m)\right)$. Our calculations are based on the following alternative construction of the uniform random mapping digraph $G_{n}$. 
Suppose that $\hat{L}_{n}$ is a discrete random variable such that $\hat{L}_{n}$ has the same distribution as $L_{n}$ (denoted $\hat{L}_{n} \sim L_{n}$ ), where $L_{n}$ is the number of cyclic vertices in a uniform random mapping. Given $\hat{L}_{n}=\ell$, let $\mathcal{A}_{\ell}$ denote a uniform random subset of size $\ell$ from the vertices $V_{n}$ (i.e. all subsets of size $\ell$ are equally likely). Given $\mathcal{A}_{\ell}=A \subseteq V_{n}$, let $\mathcal{F}_{n}(A)$ denote the uniform random rooted forest on the vertices $V_{n}$, where $A$ is the set of roots, and direct the edges in the trees of $\mathcal{F}_{n}(A)$ such that any path from a vertex to a root is directed towards the root. Finally, let $\sigma_{A}$ denote a uniform random permutation on the set of root vertices $A$ which is independent of $\mathcal{F}_{n}(A)$. We form the directed graph $\hat{G}_{n}$ from the rooted forest $\mathcal{F}_{n}(A)$ by adding a directed edge from $i \in A$ to $j \in A$ if $\sigma_{A}(i)=j$, and we let $\hat{T}_{n}$ denote the random mapping which is represented by $\hat{G}_{n}$. Then since $\hat{L}_{n} \sim L_{n}$, we have $\hat{G}_{n} \sim G_{n}$ and $\hat{T}_{n} \sim T_{n}$.

It follows from the alternative construction of $G_{n}$ described above and the definition of the trimmed mapping digraph $G_{n}^{m}$, that the conditional distribution of $t_{n}(m)$ given $L_{n}$, the number of cyclic vertices in $G_{n}$, can be determined by considering a uniform random forest from which $m$ edges are deleted. Specifically, let $\mathcal{F}_{n, \ell}$ denote a uniformly distributed random element from the set of all forests on $n$ labelled vertices which consists of $\ell$ trees rooted at $\ell$ given vertices. For $1 \leq m \leq n-\ell$, let $\mathcal{F}_{n, \ell}(m)$ denote the forest obtained from $\mathcal{F}_{n, \ell}$ by deleting at random $m$ edges in $\mathcal{F}_{n, \ell}$ such that any subset of $m$ edges is equally likely to be deleted. We note that $\mathcal{F}_{n, \ell}(m)$ is a random forest on $n$ vertices consisting of $\ell+m$ trees rooted at $\ell+m$ vertices. The trees in $\mathcal{F}_{n, \ell}(m)$ can be classified as either 'old' or 'new' trees: there are $\ell$ 'old' trees rooted at the original $\ell$ roots of the forest $\mathcal{F}_{n, \ell}$ and $m$ 'new' trees which were created when the $m$ random edges of $\mathcal{F}_{n, \ell}$ were deleted. Let $f_{n, \ell}(m)$ denote the total number of vertices in the $m$ 'new' trees in the forest $\mathcal{F}_{n, \ell}(m)$. Then it is a straightforward consequence of the alternative construction of $G_{n}$ and the definition of $G_{n}^{m}$ and $\mathcal{F}_{n, \ell}(m)$ that for $1 \leq m \leq n-\ell$

$$
\operatorname{Pr}\left\{t_{n}(m)=m+t \mid L_{n}=\ell\right\}=\operatorname{Pr}\left\{f_{n, \ell}(m)=m+t\right\} .
$$

The distribution of $f_{n, \ell}(m)$ in this case is given by

Lemma 1. For $1 \leq m \leq n-\ell$ and $0 \leq t \leq n-\ell-m$,

$$
\operatorname{Pr}\left\{f_{n, \ell}(m)=m+t\right\}=\left(\begin{array}{c}
n-\ell-m \\
t
\end{array}\right) \frac{m}{n}\left(\frac{m+t}{n}\right)^{t-1}\left(1-\frac{m+t}{n}\right)^{n-\ell-m-t} .
$$


Proof. Without loss of generality, we may assume that the $\ell$ given roots of the random forest $\mathcal{F}_{n, \ell}$ are labelled $1,2, \ldots, \ell$ and that the $m$ roots of the 'new' trees created by cutting $m$ edges of $\mathcal{F}_{n, \ell}$ are labelled $\ell+1, \ell+2, \ldots \ell+m$ (i.e. cutting the $m$ edges of $\mathcal{F}_{n, \ell}$ which results in making the vertices $\ell+1, \ell+$ $2, \ldots, \ell+m$ roots of the 'new' trees is statistically equivalent to cutting $m$ edges of $\mathcal{F}_{n, \ell}$ at random). It follows that

$$
\operatorname{Pr}\left\{f_{n, \ell}(m)=m+t\right\}=\frac{a_{n, \ell, m}(t)}{\ell n^{n-\ell-1}}
$$

where $\ell n^{n-\ell-1}$ equals the number of forests on $n$ labelled vertices with $\ell$ trees rooted at the vertices $1,2, \ldots, \ell$ and $a_{n, \ell, m}(t)$ equals the number of forests on $n$ labelled vertices with $\ell$ trees rooted at $1,2, \ldots, \ell$ such that by making the vertices $\ell+1, \ldots, \ell+m$ roots of 'new' trees we obtain $f_{n, \ell}(m)=m+t$. We determine $a_{n, \ell, m}(t)$ by 'reversing' the cutting process.

First, we note that it follows from Cayley's formula that the number of forests on $n$ vertices with $\ell+m$ trees rooted at $1,2, \ldots, \ell+m$ such that $f_{n, \ell}(m)=m+t$ is equal to $\left(\begin{array}{c}n-\ell-m \\ t\end{array}\right) m(m+t)^{t-1} \ell(n-m-t)^{n-\ell-m-t-1}$. Next suppose that $F_{n, \ell+m}$ is a fixed forest on $n$ vertices with $\ell+m$ trees rooted at the vertices $1,2, \ldots, \ell+m$, and, for $1 \leq i \leq \ell+m$, let $t_{i}$ denote the number of vertices in the tree rooted at vertex $i$ in $F_{n, \ell+m}$. In addition, suppose that $\sum_{i=\ell+1}^{\ell+m} t_{i}=m+t$. Then we need to count the number of ways that the root vertices $\ell+1, \ell+2, \ldots, \ell+m$ can be mapped to vertices in $V_{n}$ such that the resulting digraph is still a forest with $\ell$ trees rooted at the vertices $1,2, \ldots, \ell$. Our counting argument is based on Burtin's Lemma (see $[10,1]$ ) in the version given by Ross in [41]. Similar arguments were also used by Jaworski in the study of non-uniform random mappings with independent choices of images and related forests (see e.g. [24], [25], [26]) and by Hansen (see [18]) in the study of optimal directed spanning trees. More recently, this method has been ingeniously used and developed in many papers by Pitman, e.g., in the study of coalescent random forests [38].

Fact 1. (Burtin's Lemma) Suppose that $X_{1}, X_{2}, \ldots, X_{m}$ are i.i.d. random variables such that

$$
\operatorname{Pr}\left\{X_{1}=j\right\}=\lambda_{j} \quad j=0,1, \ldots, m \quad \text { and } \quad \sum_{j=0}^{m} \lambda_{j}=1 .
$$

Let $G$ be the random digraph on the vertices $0,1, \ldots, m$ obtained by constructing a directed edge from $i$ to $j$ if $X_{i}=j$. Then $\operatorname{Pr}\{G$ is connected $\}=\lambda_{0}$. 
To apply Burtin's Lemma, we begin by randomizing the 'choices' made by the root vertices $\ell+1, \ell+2, \ldots ., \ell+m$. Specifically, let $Y_{1}, Y_{2}, \ldots, Y_{m}$ be a sequence of i.i.d. variables such that $\operatorname{Pr}\left\{Y_{i}=j\right\}=\frac{1}{n}$ where $1 \leq i \leq m$ and $1 \leq j \leq n$. The choices made by the vertices $\ell+1, \ell+2, \ldots, \ell+m$ are determined as follows: vertex $\ell+1 \leq k \leq \ell+m$ is mapped to vertex $j$ if and only if $Y_{k-\ell}=j$. Let $G\left(F_{n, \ell+m}\right)$ denote the random digraph on $n$ vertices that is obtained after mapping the roots $\ell+1, \ell+2, \ldots, \ell+m$ into $V_{n}$ and let $\mathcal{B}_{n, \ell+m}$ denote the event that $G\left(F_{n, \ell+m}\right)$ is a forest on $n$ vertices with $\ell$ rooted trees. Then the number of ways to map the root vertices $\ell+1, \ldots, \ell+m$ into $V_{n}$ such that $G\left(F_{n, \ell+m}\right)$ is a forest on $n$ vertices with $\ell$ roots is exactly $n^{m} \times \operatorname{Pr}\left\{\mathcal{B}_{n, \ell+m}\right\}$.

We appeal to Burtin's Lemma to compute $\operatorname{Pr}\left\{\mathcal{B}_{n, \ell+m}\right\}$. To apply the lemma, we define a random digraph $\hat{G}\left(F_{n, \ell+m}\right)$ on $m+1$ vertices, labelled $0,1, \ldots, m$, which is associated with the random digraph $G\left(F_{n, \ell+m}\right)$. Specifically, for $1 \leq i \leq m$ and $1 \leq j \leq m$, there is a directed edge from vertex $i$ to vertex $j$ in $\hat{G}\left(F_{n, \ell+m}\right)$ if and only if in the random digraph $G\left(F_{n, \ell+m}\right)$ the vertex $\ell+i$ is mapped to a vertex in the tree rooted at $\ell+j$ in the forest $F_{n, \ell+m}$, and there is a directed edge in $\hat{G}\left(F_{n, \ell+m}\right)$ from the vertex $i$ to the special vertex 0 if and only if in $G\left(F_{n, \ell+m}\right)$ the vertex $\ell+i$ is mapped to a vertex in the sub-forest of $F_{n, \ell+m}$ which is rooted at the vertices $1,2, \ldots, \ell$. Now recall that the tree sizes $t_{\ell+1}, t_{\ell+2}, \ldots, t_{\ell+m}$ satisfy the constraint $\sum_{i=1}^{m} t_{\ell+i}=m+t$. So it follows from the definition of $\hat{G}\left(F_{n, \ell+m}\right)$, that $\hat{G}\left(F_{n, \ell+m}\right)$ has the same distribution as the graph $G$ described in Fact 1, where the distribution of the i.i.d. variables $X_{1}, X_{2}, \ldots, X_{m}$ is given by

$$
\operatorname{Pr}\left\{X_{1}=j\right\}=\frac{t_{\ell+j}}{n} \quad \text { for } 1 \leq j \leq m \quad \text { and } \operatorname{Pr}\left\{X_{1}=0\right\}=\frac{n-m-t}{n} .
$$

So it follows from Fact 1, that

$$
\operatorname{Pr}\left\{\hat{G}\left(F_{n, \ell+m}\right) \text { is connected }\right\}=\frac{n-t-m}{n} .
$$

We note that since vertex 0 has out-degree 0 , the digraph $\hat{G}\left(F_{n, \ell+m}\right)$ is connected if and only if $\hat{G}\left(F_{n, \ell+m}\right)$ is a tree rooted at vertex 0 (since any component of $\hat{G}\left(F_{n, \ell+m}\right)$ which contains a cycle cannot contain the vertex 0 ). Furthermore, the digraph $\hat{G}\left(F_{n, \ell+m}\right)$ is a tree rooted at vertex 0 if and only if $G\left(F_{n, \ell+m}\right)$ is a forest rooted at the vertices $1,2, . ., \ell$. So we have

$$
\operatorname{Pr}\left\{\mathcal{B}_{n, \ell+m}\right\}=\operatorname{Pr}\left\{\hat{G}\left(F_{n, \ell+m}\right) \text { is connected }\right\}=\frac{n-t-m}{n},
$$


and this equality holds for any forest $F_{n, \ell+m}$ such that the tree sizes $t_{1}, \ldots, t_{\ell}$ satisfy the constraint $\sum_{i=1}^{\ell} t_{i}=n-m-t$. So, for any forest $F_{n, \ell+m}$ such that $\sum_{i=1}^{\ell} t_{i}=n-m-t$, the number of ways to map the root vertices $\ell+1, \ldots, \ell+m$ into $V_{n}$ such that $G\left(F_{n, \ell+m}\right)$ is a forest on $V_{n}$ vertices with $\ell$ roots is equal to

$$
n^{m} \times \operatorname{Pr}\left\{\mathcal{B}_{n, \ell+m}\right\}=\frac{n^{m}(n-m-t)}{n} .
$$

It follows that

$a_{n, \ell, m}(t)=n^{m-1}(n-m-t)\left(\begin{array}{c}n-\ell-m \\ t\end{array}\right) m(m+t)^{t-1} \ell(n-m-t)^{n-\ell-m-t-1}$,

and hence we obtain the result.

Remark. We note that for $m$ and $\ell$ such that $1 \leq m \leq n-\ell$, we can re-parametrize the right-hand side of (2.2) by setting $p=\frac{m}{n}, \phi=\frac{1}{n}$ and $N=n-\ell-m$ to obtain

$$
\operatorname{Pr}\left\{t_{n}(m)=m+t \mid L_{n}=\ell\right\}=\left(\begin{array}{c}
N \\
t
\end{array}\right) p(p+\phi t)^{t-1}(1-p-\phi t)^{N-t}
$$

for $0 \leq t \leq N$. So the conditional distribution of $t_{n}(m)-m$ given $L_{n}=\ell$ is a quasi-binomial distribution (QBD I) ([31, 29]). In Section 4 we need the following stronger version of a local limit theorem for QBD I given in [29].

Fact 2. Fix $0<\lambda<\xi<\infty$ and suppose that $\lambda<\alpha<\xi$ and $\ell=\alpha \sqrt{n}$. Also, suppose that $m=\beta \sqrt{n}$ for some $\beta>0$ fixed and let $N(\ell)=n-\ell-m$. Then for $t=\gamma N(\ell)$, where $0<\delta_{0}<\gamma<1-\delta_{0}<1$,

$$
\begin{gathered}
\operatorname{Pr}\left\{t_{n}(m)=m+t \mid L_{n}=\ell\right\}= \\
\frac{1}{N(\ell)} \frac{\beta}{\sqrt{2 \pi \gamma^{3}(1-\gamma)}} \exp \left\{-\frac{(\alpha \gamma-\beta(1-\gamma))^{2}}{2 \gamma(1-\gamma)}\right\}\left(1+\Delta_{0}\left(n, \lambda, \xi, \delta_{0}, \beta\right)\right)
\end{gathered}
$$

for all sufficiently large $n$, where $\left|\Delta_{0}\left(n, \lambda, \xi, \delta_{0}, \beta\right)\right| \leq C\left(\lambda, \xi, \delta_{0}, \beta\right) / \sqrt{n}$ and $C\left(\lambda, \xi, \delta_{0}, \beta\right)$ is a constant which depends only on $\lambda, \xi, \delta_{0}$ and $\beta$.

The proof of Fact 2 follows from (2.3) by an straightforward application of the deMoivre-Laplace local limit theorem (see Feller [14]). Also, it is not difficult to check that

$$
\int_{0}^{1} \frac{\beta}{\sqrt{2 \pi \gamma^{3}(1-\gamma)}} \exp \left\{-\frac{(\alpha \gamma-\beta(1-\gamma))^{2}}{2 \gamma(1-\gamma)}\right\} \mathrm{d} \gamma=1 .
$$




\section{Characterization of the $\mathcal{P} \mathcal{D}(\theta)$ distribution}

The Poisson-Dirichlet distribution, introduced by Kingman [32], has been studied extensively in the literature (see, for example, Donnelly and Joyce [12], Perman [36], Pitman and Yor [37]). In this section we give a convenient characterization of the $\mathcal{P} \mathcal{D}(\theta)$ distribution which also yields a useful principle for establishing convergence in distribution to the $\mathcal{P} \mathcal{D}(\theta)$ distribution on the simplex $\nabla$. In particular, we construct a sequence of random variables $Q_{1}, Q_{2}, \ldots$ such that $\left(Q_{1}, Q_{2}, \ldots\right) \in \nabla$ and the measure induced on $\nabla$ by the joint distribution of $\left(Q_{1}, Q_{2}, ..\right)$ is the $\mathcal{P} \mathcal{D}(\theta)$ distribution.

The construction of the variables $\left(Q_{1}, Q_{2}, \ldots\right)$ is based on a sequence, $Z_{1}, Z_{2}, \ldots$, of i.i.d. random variables such that $Z_{1} \sim \operatorname{Beta}(\theta)$ with parameter $\theta>0$ and density given by

$$
f(z)=\theta(1-z)^{\theta-1}
$$

on the unit interval $(0,1)$. We define a transformation $\phi$ of the sequence $\left(Z_{1}, Z_{2}, \ldots\right)$ such that $\phi\left(Z_{1}, Z_{2}, \ldots\right)=\left(W_{1}, W_{2}, \ldots\right)$ where $W_{1}=Z_{1}$ and $W_{k}=Z_{k}\left(1-Z_{1}\right)\left(1-Z_{2}\right) \cdots\left(1-Z_{k-1}\right)$ for $k>1$, and observe that $\left(W_{1}, W_{2}, \ldots\right) \in \tilde{\nabla}=\left\{\left\{x_{i}\right\}: x_{i} \geq 0, \sum x_{i} \leq 1\right\}$. The distribution of $\left(W_{1}, W_{2}, \ldots\right)$ is called the $\operatorname{GEM}(\theta)$ distribution, after Griffiths, Engen and McCloskey. Finally, we define the map $\psi: \tilde{\nabla} \rightarrow \nabla$ such that $\left(\psi\left\{x_{i}\right\}\right)_{k}$ is the $k$ th largest term in the sequence $\left\{x_{i}\right\} \in \tilde{\nabla}$; then the random sequence $\left(Q_{1}, Q_{2}, Q_{3}, \ldots\right) \equiv \psi \circ \phi\left(Z_{1}, Z_{2}, \ldots\right) \in \nabla$ has a $\mathcal{P} \mathcal{D}(\theta)$ distribution.

As a consequence of the construction described above, we have the following convergence principle: suppose that $\left(Z_{1}(n), Z_{2}(n), \ldots\right)$ is an array of random variables such that the joint distribution of $\left(Z_{1}(n), Z_{2}(n), \ldots\right)$ converges to the joint distribution of the variables $\left(Z_{1}, Z_{2}, \ldots\right)$ as $n \rightarrow \infty$. Then the joint distribution of the random sequence $\psi \circ \phi\left(Z_{1}(n), Z_{2}(n), \ldots\right)=\left(Q_{1}(n), Q_{2}(n), \ldots\right)$ converges in distribution to the $\mathcal{P} \mathcal{D}(\theta)$ distribution on $\nabla$ as $n \rightarrow \infty$ (for further details, see Hansen [11] and the references therein). To see how this convergence principle can be applied to random mappings and trimmed random mappings, we introduce some additional notation.

First, given $G_{n}$, the directed random graph which represents the random mapping $\mathcal{T}_{n}$, let $\mathcal{K}_{n}^{(1)}$ denote the component in $G_{n}$ which contains vertex labelled by 1 . If $\mathcal{K}_{n}^{(1)} \neq G_{n}$, then let $\mathcal{K}_{n}^{(2)}$ denote the component in $G_{n} \backslash \mathcal{K}_{n}^{(1)}$ which contains the vertex with smallest label; otherwise, set $\mathcal{K}_{n}^{(2)}=\emptyset$. For $i>2$, we define $\mathcal{K}_{n}^{(i)}$ iteratively: If $G_{n} \backslash\left(\mathcal{K}_{n}^{(1)} \cup \ldots \cup \mathcal{K}_{n}^{(i-1)}\right) \neq \emptyset$, then let $\mathcal{K}_{n}^{(i)}$ 
denote the component in $G_{n} \backslash\left(\mathcal{K}_{n}^{(1)} \cup \ldots \cup \mathcal{K}_{n}^{(i-1)}\right)$ which contains the vertex with smallest label; otherwise, set $\mathcal{K}_{n}^{(i)}=\emptyset$. For $i \geq 1$, let $k_{n}^{(i)}=\left|\mathcal{K}_{n}^{(i)}\right|$ and define the sequence $\left(z_{n}^{(1)}, z_{n}^{(2)}, \ldots\right)$ by

$$
z_{n}^{(1)}=\frac{k_{n}^{(1)}}{n}, z_{n}^{(2)}=\frac{k_{n}^{(2)}}{n-k_{n}^{(1)}}, \ldots, z_{n}^{(i)}=\frac{k_{n}^{(i)}}{n-k_{n}^{(1)}-k_{n}^{(2)}-\ldots-k_{n}^{(i-1)}}, \ldots
$$

where $z_{n}^{(i)}=0$ if $n-k_{n}^{(1)}-k_{n}^{(2)}-\ldots-k_{n}^{(i-1)}=0$. For $i \geq 1$, we also define $d_{n}^{(i)}$ to be the size of the $i^{\text {th }}$ largest component in $G_{n}$. It is easy to check that

$$
\psi \circ \phi\left(z_{n}^{(1)}, z_{n}^{(2)} \ldots\right)=\left(\frac{d_{n}^{(1)}}{n}, \frac{d_{n}^{(2)}}{n}, \ldots\right),
$$

Now it is well known (see [33], [9]) that for $0<a<b<1$

$$
\lim _{n \rightarrow \infty} \operatorname{Pr}\left\{a<z_{n}^{(1)} \leq b: 1 \leq i \leq k\right\}=\int_{a}^{b} \frac{d u}{2 \sqrt{1-u}} .
$$

It can also be shown by straightforward counting arguments that the conditional distribution of $k_{n}^{(j)}$ given $k_{n}^{(1)}=s_{1}, \ldots, k_{n}^{(j-1)}=s_{j-1}$ (where $j \geq 2$ and $\left.s_{1}+\ldots+s_{j}<n\right)$ is the same as the distribution of $k_{n-s_{1}-\ldots s_{j}}^{(1)}$. It follows by an induction argument that for each $j \geq 1$ and $0<a_{i}<b_{i}<1, \quad i=1,2, \ldots, j$ we have

$$
\lim _{n \rightarrow \infty} \operatorname{Pr}\left\{a_{i}<z_{n}^{(i)} \leq b_{i}: 1 \leq i \leq j\right\}=\prod_{i=1}^{j} \int_{a_{i}}^{b_{i}} \frac{d u}{2 \sqrt{1-u}} .
$$

Hence, by the convergence principle stated above, the joint distribution of the sequence $\left(d_{n}^{(1)} / n, d_{n}^{(2)} / n, \ldots\right)$ converges in distribution to the $\mathcal{P} \mathcal{D}(1 / 2)$ distribution on $\nabla$.

We can also adapt the argument given above to investigate the joint distribution of the order statistics of the normalised component sizes in $G_{n}^{m}$. Specifically, given $G_{n}^{m}$, the directed random graph which represents the trimmed random mapping $\mathcal{T}_{n}^{m}$, let $\mathcal{K}_{n}^{(1)}(m)$ denote the component in $G_{n}^{m}$ which contains the vertex with the smallest label (which may not be the vertex labelled 1$)$. If $\mathcal{K}_{n}^{(1)}(m) \neq G_{n}^{m}$, then let $\mathcal{K}_{n}^{(2)}(m)$ denote the component in $G_{n}^{m} \backslash \mathcal{K}_{n}^{(1)}(m)$ which contains the vertex with the smallest label; otherwise, set $\mathcal{K}_{n}^{(2)}(m)=\emptyset$. For $i>2$, we define $\mathcal{K}_{n}^{(i)}(m)$ iteratively: If 
$G_{n}^{m} \backslash\left(\mathcal{K}_{n}^{(1)}(m) \cup \ldots \cup \mathcal{K}_{n}^{(i-1)}(m)\right) \neq \emptyset$, then let $\mathcal{K}_{n}^{(i)}(m)$ denote the component in $G_{n} \backslash\left(\mathcal{K}_{n}^{(1)}(m) \cup \ldots \cup \mathcal{K}_{n}^{(i-1)}(m)\right)$ which contains the vertex with the smallest label; otherwise, set $\mathcal{K}_{n}^{(i)}(m)=\emptyset$. For $i \geq 1$, let $k_{n}^{(i)}(m)=\left|\mathcal{K}_{n}^{(i)}(m)\right|$ and define the sequence $\left(z_{n}^{(1)}(m), z_{n}^{(2)}(m), \ldots\right)$ by

$$
\begin{gathered}
z_{n}^{(1)}(m)=\frac{k_{n}^{(1)}(m)}{\nu_{n}(m)}, z_{n}^{(2)}(m)=\frac{k_{n}^{(2)}(m)}{\nu_{n}(m)-k_{n}^{(1)}(m)}, \ldots, \\
z_{n}^{(i)}(m)=\frac{k_{n}^{(i)}(m)}{\nu_{n}(m)-k_{n}^{(1)}(m)-k_{n}^{(2)}(m)-\ldots-k_{n}^{(i-1)}(m)}, \ldots
\end{gathered}
$$

where $z_{n}^{(i)}(m)=0$ if $\nu_{n}(m)-k_{n}^{(1)}(m)-k_{n}^{(2)}(m)-\ldots-k_{n}^{(i-1)}(m)=0$. For $i \geq 1$, we also define $d_{n}^{(i)}(m)$ to be the size of the $i^{\text {th }}$ largest component in $G_{n}^{m}$. So, as in the case of random mappings, to show that the sequence $\left(d_{n}^{(1)}(m) / n, d_{n}^{(2)}(m) / n, \ldots\right)$ converges in distribution to a $\mathcal{P} \mathcal{D}(\theta)$ distribution on $\nabla$, it is necessary and sufficient to show that for each $k \geq 1$ and $0<a_{i}<$ $b_{i}<1, \quad i=1,2, \ldots, k$, we have

$$
\lim _{n \rightarrow \infty} \operatorname{Pr}\left\{a_{i}<z_{n}^{(i)}(m) \leq b_{i}: 1 \leq i \leq k\right\}=\prod_{i=1}^{k} \int_{a_{i}}^{b_{i}} \theta(1-u)^{\theta-1} d u .
$$

\section{The asymptotic component structure of a trimmed random mapping}

In this section we show that the joint distribution of the order statistics of the normalised component sizes of $G_{n}^{m}$ converges to the Poisson-Dirichlet $(\theta)$ distribution as $n \rightarrow \infty$ when either $m=o(\sqrt{n})$ or $\sqrt{n}=o(m)$. We do not obtain a Poisson-Dirichlet limiting distribution during the 'phase transition' when $m=\beta \sqrt{n}$ and we consider this case first.

Theorem 1. Let $\beta$ be a positive real parameter and suppose that $m=\beta \sqrt{n}$. Then for any $0<a<b<1$.

$$
\lim _{n \rightarrow \infty} \operatorname{Pr}\left\{a<z_{n}^{(1)}(m) \leq b\right\}=\int_{a}^{b} f_{\beta}(x) d x
$$


where $f_{\beta}$ is a density function on the interval $(0,1)$ which is given by the integral

$$
\begin{gathered}
f_{\beta}(x)=\int_{0}^{\infty} \int_{0}^{1} \int_{0}^{1} \frac{\alpha^{2} \beta(1-y)}{2 \pi \sqrt{x(1-x)^{3}}(1-\gamma) \sqrt{\gamma^{3}}} \\
\times \exp \left\{-\frac{(x-y)^{2} \alpha^{2}}{2 x(1-x)(1-\gamma)}-\frac{(\alpha \gamma-\beta(1-\gamma))^{2}}{2 \gamma(1-\gamma)}-\frac{\alpha^{2}}{2}\right\} \mathrm{d} y \mathrm{~d} \gamma \mathrm{d} \alpha .
\end{gathered}
$$

Moreover for any $0<a<b<1$,

$$
\lim _{\beta \downarrow 0} \int_{a}^{b} f_{\beta}(x) \mathrm{d} x=\int_{a}^{b} \frac{\mathrm{d} x}{2 \sqrt{1-x}} \quad \text { and } \quad \lim _{\beta \rightarrow \infty} \int_{a}^{b} f_{\beta}(x) \mathrm{d} x=\int_{a}^{b} 1 \mathrm{~d} x .
$$

Proof. We begin by fixing $0<a<b<1$ and $\varepsilon>0$ arbitrarily small and by letting $\mathcal{A}_{1}(n, m)=\left\{a<z_{n}^{(1)}(m) \leq b\right\}$. Since $L_{n} / \sqrt{n}$ converges in distribution as $n \rightarrow \infty$, we can choose $0<\lambda(\varepsilon)<\xi(\varepsilon)<\infty$ such that $\operatorname{Pr}\left\{\lambda(\varepsilon) \sqrt{n}<L_{n}<\xi(\varepsilon) \sqrt{n}\right\}>1-\frac{\varepsilon}{2}$ for all sufficiently large $n$. Next, it follows from Fact 2 that there exists $0<\phi(\varepsilon)<\psi(\varepsilon)<\infty$ such that for $\lambda(\varepsilon) \sqrt{n}<\ell<\xi(\varepsilon) \sqrt{n}$ and all sufficiently large $n$,

$$
\operatorname{Pr}\left\{\phi(\varepsilon) N(\ell)<t_{n}(m)-m<\psi(\varepsilon) N(\ell) \mid L_{n}=\ell\right\}>1-\varepsilon / 2
$$

where $N(\ell)=n-m-\ell$. Finally, $\lim _{n \rightarrow \infty} \operatorname{Pr}\left\{L_{n}>n-m\right\}=0$ since $m=\beta \sqrt{n}$, so

$$
\lim _{n \rightarrow \infty} \operatorname{Pr}\left\{a<z_{n}^{(1)}(m) \leq b\right\}=\lim _{n \rightarrow \infty} \operatorname{Pr}\left\{\mathcal{A}_{1}(n, m), L_{n} \leq n-m\right\} .
$$

So, we have

$$
\begin{gathered}
\operatorname{Pr}\left\{\mathcal{A}_{1}(n, m), L_{n} \leq n-m\right\} \\
=\sum_{\ell=1}^{n-m} \sum_{t=0}^{n-m-l} \operatorname{Pr}\left\{\mathcal{A}_{1}(n, m) \mid L_{n}=\ell, t_{n}(m)=m+t\right\} \\
\times \operatorname{Pr}\left\{t_{n}(m)=m+t \mid L_{n}=\ell\right\} \times \operatorname{Pr}\left\{L_{n}=\ell\right\} \\
=\Sigma(\varepsilon)+\sum_{\ell>\lambda(\varepsilon) \sqrt{n}}^{\xi(\varepsilon) \sqrt{n}} \sum_{t>\phi(\varepsilon) N(\ell)}^{\psi(\varepsilon) N(\ell)} \operatorname{Pr}\left\{\mathcal{A}_{1}(n, m) \mid L_{n}=\ell, t_{n}(m)=m+t\right\} \\
\times \operatorname{Pr}\left\{t_{n}(m)=m+t \mid L_{n}=\ell\right\} \times \operatorname{Pr}\left\{L_{n}=\ell\right\}
\end{gathered}
$$

where $|\Sigma(\varepsilon)|<\varepsilon$. 
Now suppose that $\lambda(\varepsilon) \sqrt{n} \leq \ell \leq \xi(\varepsilon) \sqrt{n}$ and $\phi(\varepsilon) N(\ell) \leq t \leq \psi(\varepsilon) N(\ell)$ and let $\nu=n-m-t$. Then, given $L_{n}=\ell$ and $t_{n}(m)-m=t$ (i.e. $\left.\nu_{n}(m)=n-m-t=\nu\right)$, we can re-label the vertices in $\mathcal{V}_{n}(m)$, the vertex set of $G_{n}^{m}$, according to their natural order by $1,2, . ., \nu$. It is straightforward to check using arguments from Section 2 that the conditional distribution of $G_{n}^{m}$ given $L_{n}=\ell$ and $t_{n}(m)-m=t$ corresponds to the conditional distribution of $G_{\nu}$ given $L_{\nu}=\ell$. In particular,

$$
\operatorname{Pr}\left\{\mathcal{A}_{1}(n, m) \mid L_{n}=\ell, t_{n}(m)-m=t\right\}=\operatorname{Pr}\left\{a<\frac{k_{\nu}^{(1)}}{\nu} \leq b \mid L_{\nu}=\ell\right\} .
$$

To approximate the right-hand side of (4.4) we appeal to a local limit theorem (see [21], Theorem 3): for any $a<x \leq b, \rho>0$ and all sufficiently large $\nu$

$$
\begin{gathered}
\operatorname{Pr}\left\{\frac{k_{\nu}^{(1)}}{\nu}=x \mid \frac{L_{\nu}}{\sqrt{\nu}}=\rho\right\} \\
=\frac{1}{\nu} \frac{\rho}{\sqrt{2 \pi x(1-x)^{3}}} \int_{0}^{1}(1-y) \exp \left\{-\frac{(x-y)^{2} \rho^{2}}{2 x(1-x)}\right\} \mathrm{d} y(1+\Delta(\nu, \rho, x))
\end{gathered}
$$

where $|\Delta(\nu, \rho, x)|<C\left(\frac{\rho^{3} \vee 1}{\min \left(\delta^{5}, \rho\right)}\right) \exp \left(\frac{\rho^{2}}{\delta^{2}}\right) \frac{1}{\sqrt{\nu}}$ and $C$ is a constant which is independent of $\nu, \rho$, and $\delta \equiv \min (a, 1-b)$.

Now suppose that $\ell=\alpha \sqrt{n}$ for some $\lambda(\varepsilon) \leq \alpha \leq \xi(\varepsilon)$ and $\phi(\varepsilon) N(\ell) \leq t \leq$ $\psi(\varepsilon) N(\ell)$. Then $t=\gamma N(\ell)$ for some $\phi(\varepsilon) \leq \gamma \leq \psi(\varepsilon)$ and $\nu=n-t-m=$ $n-m-\gamma N(\ell)$. We have from (4.4)

$\operatorname{Pr}\left\{\mathcal{A}_{1}(n, m) \mid L_{n}=\ell, t_{n}(m)=m+t\right\}=\operatorname{Pr}\left\{a<\frac{k_{\nu}^{(1)}}{\nu} \leq b \mid L_{\nu}=\alpha \sqrt{\frac{n}{\nu}} \sqrt{\nu}\right\}$,

and we note that

$$
\sqrt{\frac{n}{\nu}}=\sqrt{\frac{n}{n-m-\gamma N}}=\frac{1}{\sqrt{1-\gamma+O(1 / \sqrt{n})}} \sim \frac{1}{\sqrt{1-\gamma}} .
$$

It follows from (4.5-4.7) that

$$
\begin{aligned}
& \operatorname{Pr}\left\{\mathcal{A}_{1}(n, m) \mid L_{n}=\ell, t_{n}(m)=m+t\right\} \\
& =\int_{a}^{b} \frac{\alpha}{\sqrt{2 \pi x(1-x)^{3}(1-\gamma)}} \int_{0}^{1}(1-y) \exp \left\{-\frac{(x-y)^{2} \alpha^{2}}{2 x(1-x)(1-\gamma)}\right\} \mathrm{d} y \mathrm{~d} x \\
& \quad \times(1+\tilde{\Delta}(\nu, \alpha, \gamma, \delta))
\end{aligned}
$$


where

$$
|\tilde{\Delta}(\nu, \alpha, \gamma, \delta)| \leq \mathcal{C}(\lambda(\varepsilon), \xi(\varepsilon), \phi(\varepsilon), \psi(\varepsilon), \delta) / \sqrt{n}
$$

and $\mathcal{C}(\lambda(\varepsilon), \xi(\varepsilon), \phi(\varepsilon), \psi(\varepsilon), \delta)$ is a constant which depends only on $\lambda(\varepsilon), \xi(\varepsilon)$, $\phi(\varepsilon), \psi(\varepsilon)$, and $\delta$. It is also known (see [22]) that for $\ell=\alpha \sqrt{n}$ where $\lambda(\varepsilon)<\alpha<\xi(\varepsilon)$

$$
\operatorname{Pr}\left\{L_{n}=\ell\right\}=\left(\begin{array}{l}
n \\
\ell
\end{array}\right) \frac{\ell !\left(\ell n^{n-\ell-1}\right)}{n^{n}}=\frac{1}{\sqrt{n}} \alpha e^{-\alpha^{2} / 2}\left(1+\Delta^{\prime}(n, \alpha)\right)
$$

where $\left|\Delta^{\prime}(n, \alpha)\right| \leq \mathcal{C}^{\prime}(\lambda(\varepsilon), \xi(\varepsilon)) / \sqrt{n}$ and $\mathcal{C}^{\prime}(\lambda(\varepsilon), \xi(\varepsilon))$ is a constant which depends only on $\lambda(\varepsilon)$ and $\xi(\varepsilon)$. Finally, it follows from Fact 2, (4.8), and (4.9) that

$$
\begin{gathered}
\sum_{\ell>\lambda(\varepsilon) \sqrt{n}}^{\xi(\varepsilon) \sqrt{n}} \sum_{t>\phi(\varepsilon) N(\ell)}^{\psi(\varepsilon) N(\ell)} \operatorname{Pr}\left\{\mathcal{A}_{1}(n, m) \mid L_{n}=\ell, t_{n}(m)=m+t\right\} \\
\times \operatorname{Pr}\left\{t_{n}(m)=m+t \mid L_{n}=\ell\right\} \times \operatorname{Pr}\left\{L_{n}=\ell\right\} \\
\sim \int_{\lambda(\varepsilon)}^{\xi(\varepsilon)} \int_{\phi(\varepsilon)}^{\psi(\varepsilon)} \int_{a}^{b} \int_{0}^{1} \frac{\alpha^{2} \beta(1-y)}{2 \pi \sqrt{x(1-x)^{3}}(1-\gamma) \sqrt{\gamma^{3}}} \\
\times \exp \left\{-\frac{(x-y)^{2} \alpha^{2}}{2 x(1-x)(1-\gamma)}-\frac{(\alpha \gamma-\beta(1-\gamma))^{2}}{2 \gamma(1-\gamma)}-\frac{\alpha^{2}}{2}\right\} \mathrm{d} y \mathrm{~d} x \mathrm{~d} \gamma \mathrm{d} \alpha .
\end{gathered}
$$

The last assertion of the theorem follows from tedious but essentially straightforward calculations which we omit.

Remark 1. Numerical calculations suggest that for $0<\beta<\infty$, the density $f_{\beta}(x)$ cannot be simplified to obtain $f_{\beta}(x)=\theta(1-x)^{\theta-1}$ for some $1 / 2<\theta<1$. This indicates that when $m=\beta \sqrt{n}$ the distribution of $z_{n}^{(1)}(m)$ does not converge to the Beta distribution on $(0,1)$ as $n \rightarrow \infty$. In this case, it follows from the characterization of the Poisson-Dirichlet distribution, that the joint distribution of the normalized order statistics, $\left(d_{n}^{(1)}(m) / \nu_{n}(m), d_{n}^{(2)}(m) / \nu_{n}(m), \ldots\right)$, does not converge to a $\mathcal{P} \mathcal{D}(\theta)$ distribution on $\nabla$. However, we can nevertheless view the limiting distribution of $z_{n}^{(1)}$ as a distribution which is parameterized by $0<\beta<\infty$ and which changes 'smoothly' from the $\operatorname{Beta}(1 / 2)$ distribution to $\operatorname{Beta}(1)$ distribution as the parameter $\beta$ goes from 0 to $\infty$. In particular, for very small values of $\beta$ the 


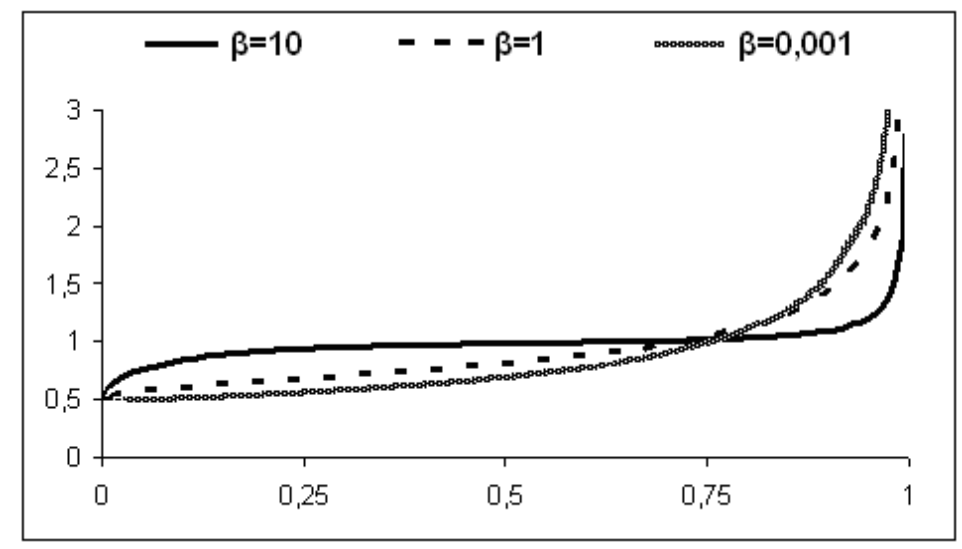

density $f_{\beta}$ is 'close' to the $\operatorname{Beta}(1 / 2)$ density on $(0,1)$, whereas for very large $\beta$, the $f_{\beta}$ is close to the $\operatorname{Beta}(1)$ density. This is illustrated in the diagram below where plots of $f_{\beta}$ are given for a small, moderate and large value of $\beta$.

Theorem 2. The joint distribution of the order statistics of the normalised component sizes of $G_{n}^{m}$ converges to the Poisson-Dirichlet(1/2) distribution as $n \rightarrow \infty$ when $m=o(\sqrt{n})$.

It follows from the convergence principle given in Section 3, that to prove the above theorem it is enough to show the following lemma.

Lemma 2. Suppose that $m=o(\sqrt{n})$, then for any $k>0$ and $0<a_{i}<b_{i}<1$, where $i=1,2, \ldots, k$, we have

$$
\lim _{n \rightarrow \infty} \operatorname{Pr}\left\{a_{i}<z_{n}^{(i)}(m) \leq b_{i}: i=1,2, \ldots, k\right\}=\prod_{i=1}^{k} \int_{a_{i}}^{b_{i}} \frac{1}{2 \sqrt{1-x}} d x
$$

where $z_{n}^{(i)}(m)=\frac{k_{n}^{(i)}(m)}{\nu_{n}(m)-k_{n}^{(1)}(m)-\ldots-k_{n}^{(i-1)}(m)}$.

Proof. To establish (4.10), we recall that for the uniform random mapping graph $G_{n}$, and for any $k \geq 1$ and $0<a_{i}<b_{i}<1, \quad i=1,2, \ldots, k$, we have

$$
\lim _{n \rightarrow \infty} \operatorname{Pr}\left\{a_{i}<z_{n}^{(i)} \leq b_{i}: 1 \leq i \leq k\right\}=\prod_{i=1}^{k} \int_{a_{i}}^{b_{i}} \frac{d u}{2 \sqrt{1-u}} .
$$


So to prove (4.10), it suffices to show that for any $\delta>0$, and any $k \geq 1$

$$
\lim _{n \rightarrow \infty} \operatorname{Pr}\left\{\max _{1 \leq i \leq k}\left|z_{n}^{(i)}-z_{n}^{(i)}(m)\right|>\delta\right\}=0 .
$$

To establish this limit, we start by fixing $\delta>0$ and $k \geq 1$, and we choose $\varepsilon>0$ arbitrarily small. Since $m=o(\sqrt{n})$, it follows from asymptotics established for quasi-binomial distribution (QBD I) (see $[10,29,30]$ ) that we can choose some bound $f(n, m)=o(n)$ such that for $n$ and $m$ large enough

$$
\operatorname{Pr}\left\{\mathcal{C}_{n}(m)\right\} \geq 1-\frac{\varepsilon}{3}
$$

where $\mathcal{C}_{n}(m)=\left\{t_{n}(m)<f(n, m)\right\}$. It also follows from (4.11) that we can fix some $0<a(\varepsilon)<b(\varepsilon)<1$ and $n_{\varepsilon}>0$ such that for all $n>n_{\varepsilon}$

$$
\operatorname{Pr}\left\{\mathcal{D}_{n}(a(\varepsilon), b(\varepsilon), k)\right\}>1-\frac{\varepsilon}{3}
$$

where

$$
\mathcal{D}_{n}(a(\varepsilon), b(\varepsilon), k)=\left\{a(\varepsilon)<z_{n}^{(i)} \leq b(\varepsilon): i=1,2, \ldots, k\right\} .
$$

Lastly, for $n>0$ and $1 \leq i \leq k$, let $v_{n}(i)$ denote the vertex with smallest label in the component $\mathcal{K}_{n}^{(i)}$ of $G_{n}$. We define the event

$$
\mathcal{E}_{n}(m, k)=\left\{v_{n}(i) \in \mathcal{K}_{n}^{(i)}(m): i=1,2, \ldots, k\right\}
$$

and we note that event $\mathcal{E}_{n}(m, k)$ implies $\mathcal{K}_{n}^{(i)}(m) \subseteq \mathcal{K}_{n}^{(i)}$ for $i=1,2, \ldots, k$.

We claim that for all sufficiently large $n$

$$
\mathcal{C}_{n}(m) \cap \mathcal{D}_{n}(a(\varepsilon), b(\varepsilon), k) \cap \mathcal{E}_{n}(m, k) \subseteq\left\{\max _{1 \leq i \leq k}\left|z_{n}^{(i)}-z_{n}^{(i)}(m)\right| \leq \delta\right\} .
$$

To see this, observe that the event $\mathcal{D}_{n}(a(\varepsilon), b(\varepsilon), k)$ implies, by induction on $i$, that for $1 \leq i \leq k$,

$$
n-k_{n}^{(1)}-\ldots-k_{n}^{(i)} \geq(1-b(\varepsilon))^{i} n .
$$

Also, the event $\mathcal{C}_{n}(m) \cap \mathcal{E}_{n}(m, k)$ implies $t_{n}(m)<f(n, m)$ and $k_{n}^{(i)}(m) \leq k_{n}^{(i)}$ for $1 \leq i \leq t$. It follows that the event $\mathcal{C}_{n}(m) \cap \mathcal{E}_{n}(m, k)$ implies, for $1 \leq i \leq k$,

$$
n-k_{n}^{(1)}-\ldots-k_{n}^{(i)}-f(n, m) \leq \nu_{n}(m)-k_{n}^{(1)}(m)-\ldots-k_{n}^{(i)}(m)
$$




$$
\leq n-k_{n}^{(1)}-\ldots-k_{n}^{(i)} .
$$

The last inequality holds since $\sum_{i=1}^{k}\left(k_{n}^{(i)}-k_{n}^{(i)}(m)\right) \leq n-\nu_{n}(m)$. It follows that the event $\mathcal{C}_{n}(m) \cap \mathcal{D}_{n}(a(\varepsilon), b(\varepsilon), k) \cap \mathcal{E}_{n}(m, k)$ implies, for $1 \leq i \leq k$,

$$
\begin{aligned}
& \left|z_{n}^{(i)}-z_{n}^{(i)}(m)\right|=\left|\frac{k_{n}^{(i)}}{n-k_{n}^{(1)}-\ldots-k_{n}^{(i-1)}}-\frac{k_{n}^{(i)}(m)}{\nu_{n}(m)-k_{n}^{(1)}(m)-\ldots-k_{n}^{(i-1)}(m)}\right| \\
& \quad \leq\left|\frac{k_{n}^{(i)}-k_{n}^{(i)}(m)}{n-k_{n}^{(1)}-\ldots-k_{n}^{(i-1)}}\right| \\
& \quad+\frac{k_{n}^{(i)}(m)}{n-k_{n}^{(1)}-\ldots-k_{n}^{(i-1)}}\left|1-\frac{n-k_{n}^{(1)}-\ldots-k_{n}^{(i-1)}}{\nu_{n}(m)-k_{n}^{(1)}(m)-\ldots-k_{n}^{(i-1)}(m)}\right| \\
& \leq \frac{t_{n}(m)}{(1-b(\varepsilon))^{i-1} n}+z_{n}^{(i)}\left|1-\frac{n-k_{n}^{(1)}-\ldots-k_{n}^{(i-1)}}{n-k_{n}^{(1)}-\ldots-k_{n}^{(i-1)}-f(n, m)}\right| \leq C \frac{f(n, m)}{(1-b(\varepsilon))^{k} n}
\end{aligned}
$$

where $C>0$ is a constant which does not depend on $n$. Since $f(n, m)=o(n)$, (4.14) holds for all sufficiently large $n$. Therefore, it suffices to show that for all large $n$, we have

$$
\operatorname{Pr}\left\{\mathcal{C}_{n}(m) \cap \mathcal{D}_{n}(a(\varepsilon), b(\varepsilon), k) \cap \mathcal{E}_{n}(m, k)\right\}>1-\varepsilon .
$$

To obtain this bound, we begin by noting that if $t_{n}(m)<f(n, m)$ and if $k_{n}^{(i)}=k_{i}$ for $1 \leq i \leq k$ such that $a(\varepsilon)<z_{n}^{(i)}<b(\varepsilon)$ for $1 \leq i \leq k$, then

$$
\begin{gathered}
\operatorname{Pr}\left\{v_{n}(i) \in \mathcal{K}_{n}^{(i)}(m) \text { for } 1 \leq i \leq k \mid t_{n}(m)<f(n, m), k_{n}^{(i)}=k_{i}, 1 \leq i \leq k\right\} \\
=\prod_{i=1}^{k} \operatorname{Pr}\left\{v_{n}(i) \in \mathcal{K}_{n}^{(i)}(m) \mid t_{n}(m)<f(n, m), k_{n}^{(i)}=k_{i}, 1 \leq i \leq k\right\} \\
\geq \prod_{i=1}^{k} \frac{k_{i}-f(n, m)}{k_{i}} \geq\left(1-\frac{f(n, m)}{a(\varepsilon)(1-b(\varepsilon))^{k} n}\right)^{k} .
\end{gathered}
$$

The last inequality follows since the event $\mathcal{D}_{n}(a(\varepsilon), b(\varepsilon), k)$ implies $k_{i} \geq$ $a(\varepsilon)(1-b(\varepsilon))^{i-1} n>a(\varepsilon)(1-b(\varepsilon))^{k} n$. So it follows that for all sufficiently large $n$, we have

$$
\operatorname{Pr}\left\{\mathcal{E}_{n}(m, k) \mid \mathcal{C}_{n}(m) \cap \mathcal{D}_{n}(a(\varepsilon), b(\varepsilon), k)\right\}>1-\frac{\varepsilon}{3} .
$$


Combining this inequality with (4.12) and (4.13), we obtain

$$
\operatorname{Pr}\left(\mathcal{C}_{n}(m) \cap \mathcal{D}_{n}(a(\varepsilon), b(\varepsilon), k) \cap \mathcal{E}_{n}(m, k)\right)>1-\varepsilon .
$$

It follows from this inequality and (4.14) that

$$
\limsup _{n \rightarrow \infty} \operatorname{Pr}\left\{\max _{1 \leq i \leq k}\left|z_{n}^{(i)}-z_{n}^{(i)}(m)\right|>\delta\right\}<\varepsilon .
$$

Since $\varepsilon>0$ was arbitrary, the result follows.

Theorem 3. The joint distribution of the order statistics of the normalised component sizes of $G_{n}^{m}$ converges to the Poisson-Dirichlet(1) distribution as $n \rightarrow \infty$ when $\sqrt{n}=o(m)$.

Again from the convergence principle given in Section 3 it follows that in order to prove the above theorem it is enough to show:

Lemma 3. Suppose that $\sqrt{n}=o(m)$, then for any $k>0$ and $0<a_{i}<b_{i}<1$, where $i=1,2, \ldots, k$, we have

$$
\lim _{n \rightarrow \infty} \operatorname{Pr}\left\{a_{i}<z_{n}^{(i)}(m) \leq b_{i}: i=1,2, \ldots, t\right\}=\prod_{i=1}^{k}\left(b_{i}-a_{i}\right) .
$$

Proof. The proof is by induction on $k>0$. First, suppose that $k=1$, and fix $\varepsilon>0$, arbitrarily small, and $0<a<b<1$. Also, let $\mathcal{A}_{1}(n, m)=\{a<$ $\left.\frac{k_{n}^{(1)}(m)}{\nu_{n}(m)} \leq b\right\}$. Since $L_{n} / \sqrt{n}$ converges in distribution as $n \rightarrow \infty$ (see [33], [9]), there exists $0<\alpha(\varepsilon)<\beta(\varepsilon)<\infty$, such that for all sufficiently large $n$, we have

$$
\operatorname{Pr}\left\{\alpha(\varepsilon) \leq \frac{L_{n}}{\sqrt{n}} \leq \beta(\varepsilon)\right\}>1-\frac{\varepsilon}{2} .
$$

In addition, since $\sqrt{n}=o(m)$, we know from the asymptotics for the quasibinomial distribution $([10,29])$ that there is some bound $M(n, m)=o(n)$, and some $n_{\varepsilon}>0$ such that

$$
\operatorname{Pr}\left\{\mathcal{B}_{n}(m, \varepsilon)\right\} \geq 1-\varepsilon
$$

for all $n \geq n_{\varepsilon}$, where

$$
\mathcal{B}_{n}(m, \varepsilon)=\left\{\alpha(\varepsilon) \leq \frac{L_{n}}{\sqrt{n}} \leq \beta(\varepsilon), \nu_{n}(m) \leq M(n, m)\right\} .
$$


Now suppose that $\alpha(\varepsilon) \sqrt{n} \leq \ell \leq \beta(\varepsilon) \sqrt{n}$ and $\ell \leq \nu \leq M(n, m)$ and recall (see (4.4)) that

$$
\operatorname{Pr}\left\{\mathcal{A}_{1}(n, m) \mid \nu_{n}(m)=\nu, L_{n}=\ell\right\}=\operatorname{Pr}\left\{a<\frac{k_{\nu}^{(1)}}{\nu} \leq b \mid L_{\nu}=\ell\right\} .
$$

Furthermore, it follows from Theorem 4 in [21] that there is some uniform bound $\delta_{n} \equiv \delta(a, b, \varepsilon, n, M(n, m))$ such that $\delta_{n} \rightarrow 0$ as $n, m \rightarrow \infty$ and such that for any $\alpha(\varepsilon) \sqrt{n} \leq \ell \leq \beta(\varepsilon) \sqrt{n}, \ell \leq \nu \leq M(n, m)$, and $0<a<x \leq b<$ 1 , we have

$$
\operatorname{Pr}\left\{k_{\nu}^{(1)}=x \nu \mid L_{\nu}=\ell\right\}=\frac{1}{\nu}(1+\varepsilon(x, \ell, \nu))
$$

where $|\varepsilon(x, \ell, \nu)| \leq \delta_{n}$ (and in the case $\nu=\ell, \varepsilon(x, \ell, \ell)$ ). It follows from (4.16) and (4.17) that

$$
\operatorname{Pr}\left\{\mathcal{A}_{1}(n, m) \mid \mathcal{B}_{n}(m, \varepsilon)\right\}=b-a+\gamma(a, b, \varepsilon, n, M(n, m))
$$

where $|\gamma(a, b, \varepsilon, n, M(n, m))| \leq \delta_{n}$. Finally, since

$$
0 \leq \operatorname{Pr}\left\{\mathcal{A}_{1}(n, m)\right\}-\operatorname{Pr}\left\{\mathcal{A}_{1}(n, m), \mathcal{B}_{n}(m, \varepsilon)\right\} \leq \operatorname{Pr}\left\{\mathcal{B}_{n}^{c}(m, \varepsilon)\right\},
$$

we obtain

$$
(b-a)-\varepsilon \leq \liminf _{n \rightarrow \infty} \operatorname{Pr}\left\{\mathcal{A}_{1}(n, m)\right\} \leq \limsup _{n \rightarrow \infty} \operatorname{Pr}\left\{\mathcal{A}_{1}(n, m)\right\} \leq(b-a)+\varepsilon .
$$

The result follows for $k=1$, since $\varepsilon>0$ was arbitrary.

For simplicity, we show how the inductive step works by showing how to obtain the result for $k=2$ given that the claim holds when $k=1$. (The argument remains essentially the same for any value of $k$ but it is messier to write down). Fix $\varepsilon>0$, arbitrarily small, and, for simplicity, let $\mathcal{A}_{i} \equiv \mathcal{A}_{i}(n, m)=\left\{a_{i}<z_{n}^{(i)}(m) \leq b_{i}\right\}$ for $i=1,2$. Then it follows from (4.15) that for all $n>n_{\varepsilon}$

$$
\left|\operatorname{Pr}\left\{\mathcal{A}_{1} \cap \mathcal{A}_{2}\right\}-\operatorname{Pr}\left\{\mathcal{A}_{1} \cap \mathcal{A}_{2} \cap \mathcal{B}_{n}(m, \varepsilon)\right\}\right|<\varepsilon .
$$

Next, from the first part of the proof and (4.15) we obtain

$b_{1}-a_{1}-\varepsilon \leq \liminf _{n \rightarrow \infty} \operatorname{Pr}\left(\mathcal{A}_{1} \cap \mathcal{B}_{n}(m, \varepsilon)\right) \leq \limsup _{n \rightarrow \infty} \operatorname{Pr}\left(\mathcal{A}_{1} \cap \mathcal{B}_{n}(m, \varepsilon)\right) \leq b_{1}-a_{1}$ 
So, since

$$
\operatorname{Pr}\left\{\mathcal{A}_{1} \cap \mathcal{A}_{2} \cap \mathcal{B}_{n}(m, \varepsilon)\right\}=\operatorname{Pr}\left\{\mathcal{A}_{2} \mid \mathcal{A}_{1} \cap \mathcal{B}_{n}(m, \varepsilon)\right\} \operatorname{Pr}\left\{\mathcal{A}_{1} \cap \mathcal{B}_{n}(m, \varepsilon)\right\}
$$

it is enough to consider

$$
\begin{gathered}
\operatorname{Pr}\left\{\mathcal{A}_{2} \mid \mathcal{A}_{1} \cap \mathcal{B}_{n}(m, \varepsilon)\right\}=\operatorname{Pr}\left\{\mathcal{A}_{2} \cap \mathcal{C}_{n}(m) \mid \mathcal{A}_{1} \cap \mathcal{B}_{n}(m, \varepsilon)\right\} \\
+\operatorname{Pr}\left\{\mathcal{A}_{2} \cap \mathcal{C}_{n}^{c}(m) \mid \mathcal{A}_{1} \cap \mathcal{B}_{n}(m, \varepsilon)\right\}
\end{gathered}
$$

where

$$
\mathcal{C}_{n}(m) \equiv\left\{\ell_{n}^{(1)}(m) \leq\left(z_{n}^{(1)}(m)+\frac{\left(1-b_{1}\right)}{2}\right) L_{n}\right\}
$$

and $\ell_{n}^{(1)}(m)$ equals the number of cyclic vertices in the component $\mathcal{K}_{n}^{(1)}(m)$ in the trimmed mapping represented by $G_{n}^{m}$.

Next we need to show that

$$
\limsup _{n \rightarrow \infty} \operatorname{Pr}\left\{\mathcal{C}_{n}^{c}(m) \mid \mathcal{A}_{1} \cap \mathcal{B}_{n}(m, \varepsilon)\right\}=0 .
$$

Fix $\alpha(\varepsilon) \sqrt{n} \leq \ell \leq \beta(\varepsilon) \sqrt{n}, \quad \ell \leq \nu \leq M(n, m)$, and $k+1=x \nu$, where $a_{1}<x \leq b_{1}$, and note that these inequalities also imply that $\sqrt{\nu}=o(\ell)$ since $M(n, m)=o(n)$. It follows from the discussion preceeding (4.4) and from (4.17) that

$$
\operatorname{Pr}\left\{k_{n}^{(1)}(m)=x \nu \mid \nu_{n}(m)=\nu, L_{n}=\ell\right\}=\frac{1}{\nu}(1+\varepsilon(x, \ell, \nu))
$$

where $|\varepsilon(x, \ell, \nu)| \leq \delta_{n}$. So we have

$$
\begin{gathered}
\operatorname{Pr}\left\{\ell_{n}^{(1)}(m) \geq x \ell+\frac{\left(1-b_{1}\right) \ell}{2} \mid k_{n}^{(1)}(m)=k+1=x \nu, \nu_{n}(m)=\nu, L_{n}=\ell\right\} \\
=\frac{\operatorname{Pr}\left\{\ell_{n}^{(1)}(m) \geq x \ell+\frac{\left(1-b_{1}\right) \ell}{2}, k_{n}^{(1)}(m)=x \nu \mid \nu_{n}(m)=\nu, L_{n}=\ell\right\}}{\operatorname{Pr}\left\{k_{n}^{(1)}(m)=x \nu \mid \nu_{n}(m)=\nu, L_{n}=\ell\right\}} \\
=\nu(1+\tilde{\varepsilon}) \operatorname{Pr}\left\{\ell_{n}^{(1)}(m) \geq x \ell+\frac{\left(1-b_{1}\right) \ell}{2}, k_{n}^{(1)}(m)=x \nu \mid \nu_{n}(m)=\nu, L_{n}=\ell\right\}
\end{gathered}
$$


where $|\tilde{\varepsilon}| \equiv|\tilde{\varepsilon}(x, \ell, \nu)| \leq|\varepsilon(x, \ell, \nu)| \leq \delta_{n}$. In the case where $\nu=\ell$, we have $k_{n}^{(1)}(m)=\ell_{n}^{(1)}(m)$ and the right-hand side of (4.24) equals 0 . In the case $\ell<\nu \leq M(n, m)$, the conditional distribution of $G_{n}^{m}$ given $L_{n}=\ell$ and $\nu_{n}(m)=\nu$ corresponds to the conditional distribution of $G_{\nu}$ given $L_{\nu}=\ell$, so it follows from Corollary 1 in [21] that

$$
\begin{gathered}
\operatorname{Pr}\left\{\ell_{n}^{(1)}(m)=j, k_{n}^{(1)}(m)=x \nu \mid \nu_{n}(m)=\nu, L_{n}=\ell\right\} \\
=\operatorname{Pr}\left\{\ell_{\nu}^{(1)}=j, k_{\nu}^{(1)}=x \nu \mid L_{\nu}=\ell\right\} \\
=\left(\begin{array}{c}
\nu-\ell \\
x \nu-j
\end{array}\right) \frac{\ell-j}{\ell \nu} x^{x \nu-j}(1-x)^{\nu-\ell-x \nu+j-1} \\
=\frac{(1-j / \ell)}{\nu(1-x)}\left(\begin{array}{c}
\nu-\ell \\
x \nu-j
\end{array}\right) x^{x \nu-j}(1-x)^{\nu-\ell-x \nu+j},
\end{gathered}
$$

where $\ell_{\nu}^{(1)}$ equals the number of cyclic vertices in the component $\mathcal{K}_{\nu}^{(1)}$. Combining (4.24) and (4.25), we obtain

$$
\begin{gathered}
\operatorname{Pr}\left\{\ell_{n}^{(1)}(m) \geq x \ell+\frac{\left(1-b_{1}\right) \ell}{2} \mid k_{n}^{(1)}(m)=x \nu, \nu_{n}(m)=\nu, L_{n}=\ell\right\} \\
=(1+\tilde{\varepsilon}) \sum_{j \geq x \ell+\frac{\left(1-b_{1}\right) \ell}{2}} \frac{(1-j / \ell)}{(1-x)}\left(\begin{array}{c}
\nu-\ell \\
x \nu-j
\end{array}\right) x^{x \nu-j}(1-x)^{\nu-\ell-x \nu+j} \\
\leq(1+\tilde{\varepsilon}) \sum_{j \geq x \ell+\frac{\left(1-b_{1}\right) \ell}{2}}\left(\begin{array}{c}
\nu-\ell \\
x \nu-j
\end{array}\right) x^{x \nu-j}(1-x)^{\nu-\ell-x \nu+j} \\
=(1+\tilde{\varepsilon}) \operatorname{Pr}\left\{X(x, \ell, \nu) \geq(\nu-\ell)(1-x)+\frac{\left(1-b_{1}\right) \ell}{2}\right\}
\end{gathered}
$$

where $X(x, \ell, \nu) \sim \operatorname{Bin}(\nu-\ell, 1-x)$. Now it follows from Markov's inequality that

$$
\begin{gathered}
\operatorname{Pr}\left\{X(x, \ell, \nu) \geq(\nu-\ell)(1-x)+\frac{\left(1-b_{1}\right) \ell}{2}\right\} \\
=\operatorname{Pr}\left\{Y(x, \ell, \nu) \geq \frac{\left(1-b_{1}\right) \ell}{2 \sqrt{x(1-x)(\nu-\ell)}}\right\}
\end{gathered}
$$




$$
\begin{aligned}
& \leq \mathbf{E}(\exp (Y(x, \ell, \nu))) \exp \left(\frac{-\left(1-b_{1}\right) \ell}{2 \sqrt{x(1-x)(\nu-\ell)}}\right) \\
& \leq \mathbf{E}(\exp (Y(x, \ell, \nu))) \exp \left(\frac{-\left(1-b_{1}\right) \alpha(\varepsilon) \sqrt{n}}{2 \sqrt{x(1-x) M(n, m)}}\right)
\end{aligned}
$$

where $Y(x, \ell, \nu)=(X(x, \ell, \nu)-(\nu-\ell)(1-x)) / \sqrt{x(1-x)(\nu-\ell)}$.

We claim that there exists a positive constant $C\left(a_{1}, b_{1}\right)$ such that for $a_{1}<x \leq b_{1}, \alpha(\varepsilon) \sqrt{n} \leq \ell \leq \beta(\varepsilon) \sqrt{n}, \ell<\nu \leq M(n, m)$, and all $n \geq 1$, we have

$$
\mathbf{E}(\exp (Y(x, \ell, \nu)))<C\left(a_{1}, b_{1}\right) .
$$

To see this, for $m \geq 1$ and $a_{1}<x \leq b_{1}$, let $\tilde{X}(m, x)$ denote a $\operatorname{Bin}(m, 1-x)$ random variable and let $\tilde{Y}(m, x)=(\tilde{X}(m, x)-m(1-x)) / \sqrt{m x(1-x)}$. Then we have

$\mathbf{E}\left(\exp (\tilde{Y}(m, x))=\left(x+(1-x) \exp \left(\frac{1}{\sqrt{m x(1-x)}}\right)\right)^{m} \exp \left(\frac{-\sqrt{m(1-x)}}{\sqrt{x}}\right)\right.$.

Routine calculations show that

$$
\mathbf{E}\left(\exp (\tilde{Y}(m, x))=\exp \left(\frac{1}{2}+\frac{g(x, m)}{\sqrt{m}}\right)\right.
$$

for all $a_{1}<x \leq b_{1}$ and $m \geq 1$, where $|g(x, m)| \leq \tilde{C}\left(a_{1}, b_{1}\right)$ and $\tilde{C}\left(a_{1}, b_{1}\right)$ is a constant that depends only on $a_{1}$ and $b_{1}$. This establishes (4.26).

Substituting inequality (4.26) into the inequalities above, we obtain for $a_{1}<x \leq b_{1}, \quad \alpha(\varepsilon) \sqrt{n} \leq \ell \leq \beta(\varepsilon) \sqrt{n}, \quad \ell \leq \nu \leq M(n, m)$ and all $n \geq 1$

$$
\begin{aligned}
\operatorname{Pr}\left\{\ell_{n}^{(1)}(m)\right. & \left.\geq x \ell+\frac{\left(1-b_{1}\right) \ell}{2} \mid k_{n}^{(1)}(m)=x \nu, \nu_{n}(m)=\nu, L_{n}=\ell\right\} \\
& \leq C\left(a_{1}, b_{1}\right) \exp \left(\frac{-\left(1-b_{1}\right) \alpha(\varepsilon) \sqrt{n}}{2 \sqrt{x(1-x) M(n, m)}}\right)
\end{aligned}
$$

and (4.22) follows.

Going back to equality (4.20), we see that it is enough to consider

$$
\operatorname{Pr}\left\{\mathcal{A}_{2} \cap \mathcal{C}_{n}(m) \mid \mathcal{A}_{1} \cap \mathcal{B}_{n}(m, \varepsilon)\right\}
$$




$$
=\operatorname{Pr}\left\{\mathcal{A}_{2} \mid \mathcal{C}_{n}(m) \cap \mathcal{A}_{1} \cap \mathcal{B}_{n}(m, \varepsilon)\right\} \operatorname{Pr}\left\{\mathcal{C}_{n}(m) \mid \mathcal{A}_{1} \cap \mathcal{B}_{n}(m, \varepsilon)\right\} .
$$

Now, by (4.22), we have

$$
\lim _{n \rightarrow \infty} \operatorname{Pr}\left\{\mathcal{C}_{n}(m) \mid \mathcal{A}_{1} \cap \mathcal{B}_{n}(m, \varepsilon)\right\}=1
$$

so it is enough to consider

$$
\lim _{n \rightarrow \infty} \operatorname{Pr}\left\{\mathcal{A}_{2} \mid \mathcal{C}_{n}(m) \cap \mathcal{A}_{1} \cap \mathcal{B}_{n}(m, \varepsilon)\right\} .
$$

Suppose that $\alpha(\varepsilon) \sqrt{n} \leq \ell \leq \beta(\varepsilon) \sqrt{n}, \quad \ell \leq \nu \leq M(n, m), \quad a_{1} \nu<k+1=$ $x \nu \leq b_{1} \nu$, and $j \leq x \ell+\frac{\left(1-b_{1}\right) \ell}{2}$. Using counting arguments from Section 2 , it is straightforward to check that the conditional distribution of $k_{n}^{(2)}(m)$ given $\ell_{n}^{(1)}(m)=j, k_{n}^{(1)}(m)=x \nu, \nu_{n}(m)=\nu$, and $L_{n}=\ell$, is the same as the conditional distribution of $k_{\nu-x \nu}^{(1)}$, the size of the component in $G_{\nu-x \nu}$ which contains the vertex labelled 1 , given $L_{\nu-x \nu}=\ell-j$, and, in particular,

$$
\begin{gathered}
\operatorname{Pr}\left\{a_{2}<z_{n}^{(2)}(m) \leq b_{2} \mid \ell_{n}^{(1)}(m)=j, k_{n}^{(1)}(m)=x \nu, \nu_{n}(m)=\nu, L_{n}=\ell\right\} \\
=\operatorname{Pr}\left\{a_{2}<z_{\nu-x \nu}^{(1)} \leq b_{2} \mid L_{\nu-x \nu}=\ell-j\right\} .
\end{gathered}
$$

In this case, we also have

$$
\frac{\alpha(\varepsilon)\left(1-b_{1}\right)}{2} \sqrt{n} \leq \frac{\left(1-b_{1}\right)}{2} \ell \leq \ell-j
$$

and

$$
\nu-x \nu \leq\left(1-a_{1}\right) \nu \leq\left(1-a_{1}\right) M(n, m)=o(n),
$$

and hence $\sqrt{\nu-x \nu}=o(\ell-j)$. It follows from (4.31) and (4.32) and the asymptotic bounds obtained in Theorem 4 of [21] that there is a uniform bound $\hat{\delta}_{n} \equiv \hat{\delta}\left(a_{1}, b_{1}, a_{2}, b_{2}, \varepsilon, n, M(n, m)\right)$ such that $\hat{\delta}_{n} \rightarrow 0$ as $n, m \rightarrow \infty$ and such that for $\alpha(\varepsilon) \sqrt{n} \leq \ell \leq \beta(\varepsilon) \sqrt{n}, \quad \ell \leq \nu \leq M(n, m), \quad a_{1} \nu<k+1=$ $x \nu \leq b_{1} \nu, j \leq x \ell+\frac{\left(1-b_{1}\right) \ell}{2}$, and $0<a_{2}<u \leq b_{2}<1$, we have

$$
\operatorname{Pr}\left\{k_{\nu-x \nu}^{(1)}=u(\nu-x \nu) \mid L_{\nu-x \nu}=\ell-j\right\}=\frac{1}{\nu}(1+\hat{\varepsilon}(u, x, \nu, \ell, j))
$$

where $|\hat{\varepsilon}(u, x, \nu, \ell, j)| \leq \hat{\delta}_{n}$. It follows from (4.30) and (4.33) that

$$
\operatorname{Pr}\left\{\mathcal{A}_{2} \mid \mathcal{C}_{n}(m) \cap \mathcal{A}_{1} \cap \mathcal{B}_{n}(m, \varepsilon)\right\}=b_{2}-a_{2}+\hat{\gamma}\left(a_{1}, b_{1}, a_{2}, b_{2}, \varepsilon, n, M(n, m)\right)
$$


where $\left|\hat{\gamma}\left(a_{1}, b_{1}, a_{2}, b_{2}, \varepsilon, n, M(n, m)\right)\right| \leq \hat{\delta}_{n}$. It follows from (4.18)-(4.21), (4.27), (4.28), and (4.34) that for all sufficiently large $n$

$$
\left|\operatorname{Pr}\left\{\mathcal{A}_{1} \cap \mathcal{A}_{2}\right\}-\left(b_{1}-a_{1}\right)\left(b_{2}-a_{2}\right)\right|<3 \varepsilon .
$$

Since $\varepsilon>0$ was arbitrary, the result follows in the case $k=2$.

\section{Acknowledgements}

J.Jaworski acknowledges the generous support by the Marie Curie IntraEuropean Fellowship No. 501863 (Randigraph) within the 6th European Community Framework Programme. This publication reflects only the author's views. The Community is not liable for any use that may be made of the information contained therein.

The authors would also like to thank an anonymous referee for carefully reading the first version of this paper and for suggestions which have improved the exposition of the final version.

\section{References}

[1] Anoulova, S., Bennies, J., Lenhard, J., Metzler, D., Sung, Y., and Weber, A., Six ways of looking at Burtin's Lemma, Amer. Math. Monthly 106 (1999), No. 4, 345-351.

[2] Aldous, D., Exchangeability and related topics, Lecture Notes in Math, 1117, Springer-Verlag (1985), New York.

[3] Aldous, D., Miermont, G. and Pitman, J., Brownian Bridge Asymptotics for Random p-mappings, Electron. J. Probab. 9 (2004), 37-56.

[4] Aldous, D., Miermont, G. and Pitman, J., Weak Convergence of Random $p$-mappings and the Exploration Process of of Inhomogeneous Continuum Random Trees, Probab. Th. Related Fields 133 (2005), 1-17.

[5] Aldous, D., and Pitman, J., The standard additive coalescent, Ann. Probab. 26(4) (1998), 1703-1726. 
[6] Arratia, R. and Tavaré, S., Limit theorems for combinatorial structures via discrete process approximations, Random Structures and Algorithms 3 (1992), 321-345.

[7] Arratia, R., Stark, D. and Tavaré, S., Total variation asymptotics for Poisson process approximations of logarithmic combinatorial assemblies, Ann. Probab. 23 (1995), 1347-1388.

[8] Arratia, R., Barbour, A. D. and Tavaré, S., Limits of logarithmic combinatorial structures, Ann. Probab. 28 (2000), 1620-1644.

[9] Bollobás, B., Random Graphs, Academic Press (1985), London.

[10] Burtin, Y. D., On simple formula for random mappings and its applications, J. Appl. Probab. 17 (1980), 403-414.

[11] Chassaing, P., and Louchard, G., Phase transitions for parking blocks, Brownian excursions and coalescence, Random Structures and Algorithms 21(1) (2002), 76-119 .

[12] Donnelly, P. and Joyce, P., Continuity and weak convergence of ranked and size-biased permutations on the infinite simplex, Stochastic Process. Appl. 31 (1989), 89-103.

[13] Donnelly, P. and Grimmett, G., On the asymptotic distribution of large prime factors, Jour. of the LMS 47 (1993), 395-404.

[14] Feller, W., An Introduction to Probability Theory and its Applications, Vols I and II, 3rd edition, John Wiley and Sons (1970), New York.

[15] Hansen, J. C., A functional central limit theorem for random mappings, Ann. of Probab. 17 (1989), 317-332.

[16] Hansen, J. C. and Schmutz, E., How random is the characteristic polynomial of a random matrix? Math. Proc. Cam. Phil. Soc. 114 (1993), $507-515$.

[17] Hansen, J. C., Order statistics for decomposable combinatorial structures, Random Structures and Algorithms 5 (1994), 517-533.

[18] Hansen, J. C., Limit laws for the optimal directed tree with random costs, Combinatorics, Probability and Computing 6 (1997), 315-335. 
[19] Hansen, J. C. and Jaworski, J., Large Components of Bipartite Random Mappings, Random Structures and Algorithms 17 2000), 317-342.

[20] Hansen, J. C. and Jaworski, J., Compound Random Mappings, J. Appl. Probab. 39 (2002), 712-729.

[21] Hansen, J. C. and Jaworski, J., Random mappings with a given number of cyclic vertices, submitted

(http://www.staff.amu.edu.pl/ jaworski/) (2005).

[22] Harris, B., Probability distribution related to random mappings, Ann. Math. Statist. 31 (1960), 1045-1062.

[23] Janson, S., Random cutting and records in deterministic and random trees, Random Structures and Algorithms 29(2) (2006), 139-179.

[24] Jaworski, J., On a random mapping $\left(T ; P_{j}\right)$, J. Appl. Probab. 21 (1984), 186-191.

[25] Jaworski, J., Random Mappings, unpublished dissertation, Adam Mickiewicz University, Poznań (1985),(in Polish).

[26] Jaworski, J., Random mappings with independent choices of the images, in: Random Graphs, Vol.1, Wiley (1990), 89-101.

[27] Jaworski, J. and Łuczak, T., Cycles in a uniform graph process, Combinatorics, Probability and Computing 1 (1992), 223-239.

[28] Jaworski, J. and Mutafchiev, L., The largest connected component in a random mapping, Random Structures \&6 Algorithms 5, No. 1 (1994), 73-94.

[29] Jaworski, J., Predecessors in a random mapping, Random Structures and Algorithms 13, No.3 and No.4 (1998), 501-519.

[30] Jaworski, J., Epidemic processes on digraphs of random mappings. $J$. Appl. Probab. 36 (1999), 1-19.

[31] Johnson, N. L., Kotz, S. and Kemp, A. W., Univariate Discrete Distributions, John Wiley and Sons (1992), New York. 
[32] Kingman, J. F. C., Random discrete distributions, J. Roy. Statist. Soc. Ser. B 37 (1975), 1-22.

[33] Kolchin, V. F., Random Mappings, Optimization Software Inc. (1986), New York.

[34] Mutafchiev, L., Large components and cycles in a random mapping pattern, in: Random Graphs'87(M. Karoński, J. Jaworski, A. Ruciński, eds), Wiley (1990), New York, 189-202.

[35] Meir, A. and Moon, J. W., Cutting down random trees, J. Austral. Math. Soc. 11 (1970), 313-324.

[36] Perman, M., Order statistics for jumps of normalised subordinators, Stochastic Process. Appl. 46 (1993), 267-281.

[37] Pitman, J. and Yor, M., The two-parameter Poisson-Dirichlet distribution derived from a stable subordinator, Ann. Probab. 25 (1997), 855900.

[38] Pitman, J., Coalescent random forests, J. Combin. Theory Ser. A 85(2) (1999), 165-193.

[39] Pitman, J., Combinatorial stochastic processes, In École d'été de probabilitiés de Saint-Flour, XXXII-2002, Lecture Notes in Math. 1875 Springer-Verlag (2006), Berlin.

[40] Pittel, B., On distributions related to transitive closures of random finite mappings, Ann. Probab. 11 (1983), 428-441.

[41] Ross, S. M., A Random Graph. J. Appl. Probab. 18 (1981), 309-315.

[42] Vershik, A. M.and Schmidt, A. A., Limit measures arising in the asymptotic theory of symmetric groups. I, Theor. Probab. Appl. 22 (1977), $70-85$. 